Meta

Journal des traducteurs

Translators' Journal

\title{
La traduction dans le secteur technique ou industriel
}

\section{Richard Malo}

Volume 21, numéro 1, mars 1976

La traduction et l'entreprise

URI : https://id.erudit.org/iderudit/003546ar

DOI : https://doi.org/10.7202/003546ar

Aller au sommaire du numéro

Éditeur(s)

Les Presses de l'Université de Montréal

ISSN

0026-0452 (imprimé)

1492-1421 (numérique)

Découvrir la revue

Citer cet article

Malo, R. (1976). La traduction dans le secteur technique ou industriel. Meta, 21(1), 87-89. https://doi.org/10.7202/003546ar d'utilisation que vous pouvez consulter en ligne.

https://apropos.erudit.org/fr/usagers/politique-dutilisation/ 


\section{La traduction dans le secteur technique ou industriel}

Dans le domaine de la traduction technique, il y a d'abord le problème des cours de traduction technique donnés au niveau universitaire. Il n'existe pas actuellement de département ou de faculté qui donne uniquement un enseignement en traduction technique. Les cours techniques actuellement font partie d'un tout qui s'appelle traduction, et la place donnée aux cours de traduction technique est très limitée.

Il y a à peine quelques années encore, la traduction technique ne s'enseignait pas dans les universités. Et pourtant, s'il y a un domaine qui demande une solide préparation, c'est bien le secteur industriel et technique. Il y a donc eu absence de cours de traduction technique, maintenant il y a insuffisance. Certes les quelques cours qui se donnent présentement sont souvent excellents, et il faut en remercier certains professeurs bien éclairés qui ont su imposer de tels cours, permettant ainsi aux étudiants en traduction de connaître un peu le monde de la traduction technique.

Mais cela s'avère insuffisant et ne correspond pas aux besoins actuels du marché. Les entreprises sont de plus à la recherche de traducteurs techniques. Les services de traduction déploient des efforts inouïs pour décrocher la perle rare. Avec la venue de la Loi 22 et des programmes de francisation dans l'entreprise, la demande pour des traducteurs techniques augmente de jour en jour. La francisation des entreprises exige actuellement et exigera d'ici un an ou deux de plus en plus de traduction technique.

Que les élèves qui suivent actuellement des cours ne s'inquiètent pas, l'avenir de la traduction s'annonce très bien, surtout dans le secteur industriel et technique. Mais il faut faire comprendre aux universités qu'elles doivent augmenter le nombre de cours techniques et qu'éventuellement elles devront penser à la mise en place d'un département de la traduction technique. Non pas au détriment de la traduction générale, mais en parallèle.

L'autre aspect du problème concerne la formation technique des traducteurs. Il ne suffit pas d'avoir des départements de traduction technique pour régler le problème, il faut aussi que les traducteurs comprennent qu'une formation technique, spécialisée ou non, doit venir compléter et même parfaire la compétence du traducteur technique. On dit souvent que l'idéal en traduction technique c'est 
d'avoir un ingénieur ou un technicien qui ferait de la traduction. Mais pourquoi ne pourrait-on pas avoir des traducteurs techniciens ou ingénieurs? Cela n'est pas absurde et impensable.

En même temps, il faut que les entreprises comprennent aussi qu'elles doivent favoriser et aider le traducteur à suivre des cours techniques. D'ailleurs, les entreprises peuvent facilement organiser de tels cours et les donner à leurs traducteurs. On le fait pour des commis, des secrétaires, des vendeurs, des techniciens et des ingénieurs, pourquoi pas pour des professionnels de la traduction qui désirent se spécialiser dans le domaine technique?

Le traducteur doit s'ouvrir au monde de la technique et de l'industrie. Les entreprises doivent fournir aux traducteurs tous les outils nécessaires afin que les traductions techniques soient non seulement correctes au point de vue linguistique, mais aussi au point de vue technique. Le traducteur technique ne doit pas être qu'un spécialiste des mots, mais également du terme technique. Les entreprises ne doivent pas seulement exiger une bonne traduction, mais aussi une traduction techniquement bonne. L'un et l'autre, le traducteur et l'entreprise, doivent travailler ensemble pour réunir avec bonheur technique et traduction.

Enfin, une question banale en apparence mais fort importante : il faut que les entreprises acceptent d'une façon définitive le traducteur et surtout le traducteur technique. Cela est essentiel à la bonne marche d'une entreprise du secteur technique ou industriel. Elle doit favoriser la collaboration entre ces deux types d'ingénieurs. Elle doit comprendre que l'un est aussi important que l'autre.

L'apprentissage de la traduction technique doit donc commencer à l'université, se continuer par la formation du traducteur au sein de l'entreprise et se parfaire par la volonté du traducteur de s'initier au monde de l'industrie et de la technique.

La traduction technique prend de plus en plus d'importance dans notre monde moderne qui voit la technologie et l'industrie se développer à un rythme très rapide. Le traducteur doit évoluer avec ce monde et s'il choisit la profession de traducteur technique, il doit en suivre jusqu'à un certain point les règles du jeu. On peut certaincment fusionner le monde littéraire et le monde technique, qui si souvent s'affrontent ou se boudent, sans qu'ils perdent leur identité propre. Le secteur industriel et technique en profitera grandement et la traduction fera un pas de géant vers sa reconnaissance professionnelle et son acceptation officielle dans le monde de l'industrie. Si l'on critique souvent les entreprises pour leur incompréhension envers la traduction en général et le traducteur en particulier, n'oublions pas aussi que souvent on reproche à la traduction et au traducteur de refuser de communiquer avec le monde de l'industrie.

Il est primordial pour le traducteur technique de changer certaines habitudes de travail. La traduction technique surtout ne doit pas et ne peut pas se faire en vase clos. Si le traducteur technique veut être considéré comme un professionnel par l'entreprise, il doit démontrer une attitude professionnelle. De plus, il ne doit pas craindre de s'identifier au secteur industriel et technique par peur de perdre son identité. Au contraire, l'entreprise l'acceptera s'il peut travailler en collaboration avec des techniciens, des ingénieurs, des comptables, des commis de bureau 
ou des administrateurs. Si le traducteur se sent à l'aise dans une entreprise, un grand pas est franchi pour lui comme pour son employeur.

Egalement, le traducteur technique doit se faire un point d'honneur d'être aussi terminologue. Il est essentiel que tout traducteur technique pense à la terminologie dans le monde industriel. C'est là un outil important pour la traduction technique. Quand un traducteur possède déjà une bonne terminologie technique, son travail est facilité et son rendement nettement amélioré. Dans le domaine technique ou industriel, rien ne doit être fait à la hâte ou dans l'à-peu-près, et cela concerne d'abord et avant tout la traduction.

Je ne prétends pas offrir de solution magique, mais je désire seulement exprimer quelques idées sur un sujet que je connais bien et que j'aime. Par mes propos, je ne désire que l'amélioration du sort du traducteur et la reconnaissance d'une profession merveilleuse mais mal aimée, celle du traducteur technique. C'est plus qu'un simple désir, c'est ce que je souhaite ardemment depuis longtemps.

RichaRd MALO 